\title{
Spatially inhomogeneous ultrafast precessional magnetization reversal
}

\author{
W. K. Hiebert, L. Lagae, ${ }^{*}$ and J. De Boeck \\ IMEC, Kapeldreef 75, B-3001 Leuven, Belgium \\ (Received 16 May 2003; published 18 July 2003)
}

\begin{abstract}
Spatially resolved measurements of quasiballistic precessional magnetic switching in a microstructure are presented. A technique is introduced to suppress remanent end domains and allow a completely uniform, almost-perfect-macrospin initial state. Though the precessional response to a transverse magnetic field pulse is initially spatially uniform, and macrospin characteristics can be emulated, dephasing still occurs leading to nonuniformity and transient demagnetization. These nonuniformities are intensified when using coincident longitudinal and transverse pulses created by crossing current wires. The results have implications for the reliability of ballistic precessional switching in magnetic devices.
\end{abstract}

DOI: 10.1103/PhysRevB.68.020402

Precessional switching was considered as far back as 1960 in magnetism's first foray into the random access memory market. ${ }^{1,2}$ Intense interest has resurfaced in the current run for MRAM as the ultimate speed limit to magnetic switching has been approached. ${ }^{2,3-11}$ In conventional switching, a magnetic field is applied in the opposite direction to the magnetization (making this direction the lowest energy state) and reversal proceeds through thermal attempt processes, domain nucleation, and wall motion. By-passing the thermal and damping regimes, precessional switching relies solely on nonequilibrium dynamics as a fast in-plane magnetic field pulse applied perpendicularly to the magnetization induces a large angle precessional motion. By properly tailoring the length of the transverse field pulse, ${ }^{6,7}$ the motion can be terminated after $180^{\circ}$ of rotation, thus, effecting a magnetic switch via a large angle half precession in the socalled ballistic precessional switch. ${ }^{10}$

Precessional switching in microstructures has recently been directly observed by several groups. ${ }^{12-15}$ However, these reports, in concentrating on macrospin behavior, only showed response that was averaged over a portion of a microstructure or taken at spot locations. In order to properly probe the fundamental limits of this switching mode, spatially resolved measurements over the entire microstructure are a necessity. In particular, the uniformity of response (or lack thereof) will be a key issue in determining applicability for magnetic memory and sensor devices. Further, precessional switching induced by coincident orthogonal pulses, a mainstay in bit selection schemes for MRAM, has yet to be experimentally explored.

In this paper, we report spatially and vectorially resolved imaging of precessional switching. In the best effort to date in obtaining a perfect macrospin, end domains in the remanent state of a $20 \times 7 \mu \mathrm{m}^{2}$ elliptical microstructure, which strongly hamper coherent precessional motion, are suppressed with a novel technique. Characteristic macrospin response is emulated including vector-resolved quasi-ballistic switching and oscillatory final state dependence with pulse width. However, though the full spatial resolution shows complete uniformity of response for the initial motions, dephasing occurs over the course of the precession leading to the development of spatial deviations in the magnetization. For complex pulsing parameters, inhomogeneities become pronounced until a would-be demonstration of precessional switching induced by coincident orthogonal pulses is ultimately defeated by transient demagnetization.

The experimental data is obtained using time resolved scanning magneto-optical Kerr microscopy. ${ }^{11,12}$ Spatial resolution is diffraction limited at $\sim 0.7 \mu \mathrm{m}$; temporal resolution is jitter limited to nominally 50 ps by the delay generator. An optical micrograph of the device is shown in Fig. 1(a). The magnetic element sits on top (for optical access) of two crossing current carrying wires. Current pulses through the wires allow coincident transverse and longitudinal transient magnetic fields to be applied. The axes definitions remain throughout and the initial magnetization state always points along the positive $x$ axis. The transverse pulse $H_{y}(t)$ is directed to the right using a nominally $60 \mathrm{ps}$ risetime pulser. For solo transverse pulses, a reset pulse of $\sim+6.76 \mathrm{kA} / \mathrm{m}$ (85 Oe), $15 \mathrm{~ns}$ long is applied to set the initial state. For combination pulses, the reset is between 1.99 (25) and 2.39 (30) $\mathrm{kA} / \mathrm{m}(\mathrm{Oe})$ and is sustained for about $1 \mu \mathrm{s}$.

Given the importance of uniformity of response for magnetic applications, extensive effort is placed on creating a near-perfect experimental macrospin. An elliptical shape (an ellipsoid theoretically giving spatially uniform demagnetizing fields) is chosen for the element. The $16 \mathrm{~nm}$ magnetic film is sputtered with easy axis along the long axis of the ellipse, a photoresist pattern of $20 \times 7 \mu \mathrm{m}^{2}$ is defined on top, and ion milling is used to define the pattern. Isolated ellipses of this size such as those shown in Fig. 1(b) exhibit end domains in the remanent state. Such an initial state guarantees incoherence of precessional motion. ${ }^{8,16}$ Using a longitudinal biasing field to remove these domains is not an option for practical device applications.

To overcome this problem a novel method is used to improve the uniformity of magnetization in the remanent state. Instead of etching completely through during patterning, the magnetic film is etched partially leaving a mesa embedded in an extended magnetic layer (in this case leaving about $4 \mathrm{~nm}$ ). The completely uniform remanent state of such an element is shown in Fig. 1(c). ${ }^{17}$ Instead of being forced to remain parallel to the sample edges, the magnetization aligns with the easy axis as flux lines of the would-be free poles flow into the background (as shown in the vector image) and a flux closure process occurs in the background film (not shown). Though more tests need to be done, ${ }^{18}$ this embedding process may become relevant for devices where uniformity of a 


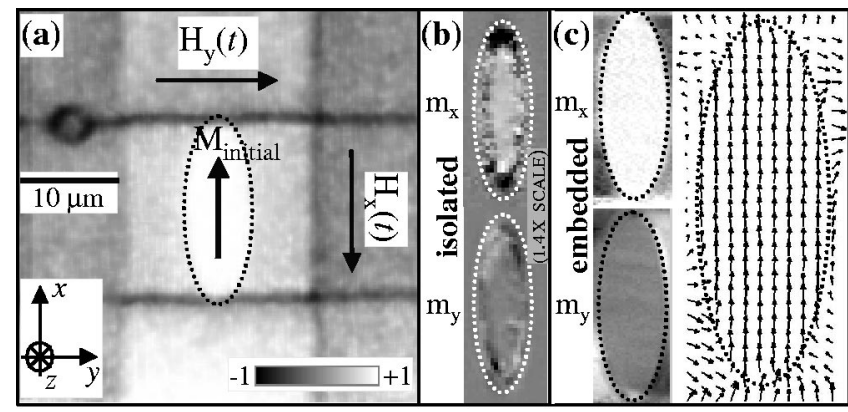

FIG. 1. Experimental geometry. (a) Optical micrograph of embedded elliptical element on top of crossing current wires with pulse field directions and axes definitions. (b) Grayscale images of $m_{x}$ and $m_{y}$ for a $12 \times 4 \mu \mathrm{m}^{2}$ isolated elliptical element showing end domains in remanence. (c) Grayscale and vector map images for the $20 \times 7 \mu \mathrm{m}^{2}$ embedded element in remanence. The closure domain process occurs in the background magnetic material leaving the elliptical mesa highly uniformly magnetized.

micron-size magnetic contact is crucial (and the cell of interest is now a stadium or rectangular shape where even less uniformity of isolated samples is expected).

The initial results are summarized in Fig. 2 where the local response of the center of the embedded ellipse is monitored as a function of transverse field pulse width. Characteristic macrospin responses discussed in earlier works are emulated in our embedded ellipse. However, the increased uniformity via embedding allows clean ballistic precessional switching to be initiated from the remanent state and in much
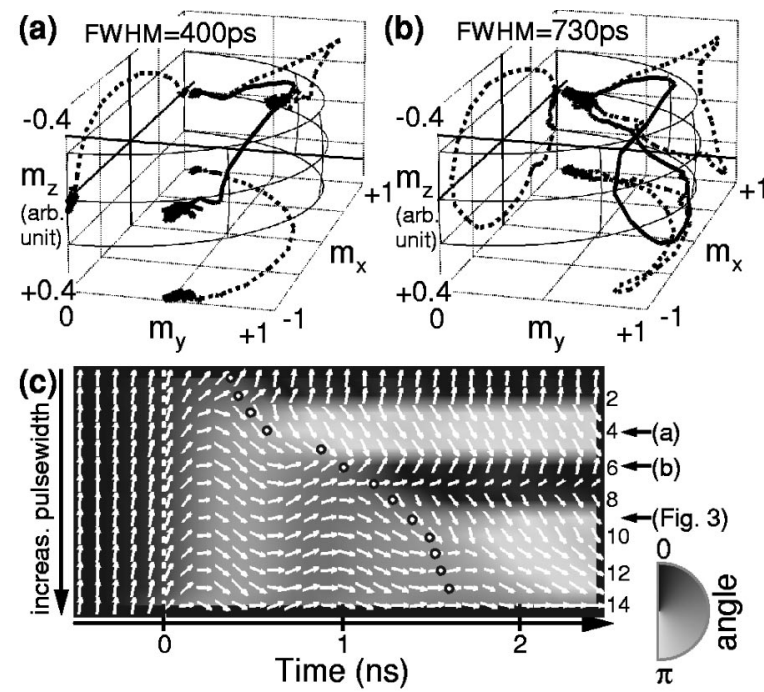

FIG. 2. Measured 3D trajectory and projections of the magnetization vector for (a) half-precession quasiballistic reversal and (b) full-precession nonreversal. The solid line is the vector tip trajectory and dotted lines are its projection onto the planes. (c) Pulse width dependence of the precessional reversal. Vectors track the in-plane direction of measured magnetization over time and the grayscale background gives the in-plane angle of simulated magnetization. Field pulses begin at the vertical dashed line and approximately end at the white dots. Increasing pulse width results first in reversal, then non reversal, then reversal again, as the hard plane is crossed once, twice, and three times, respectively. lower transverse-pulse field than observed before (even lower than the effective in-plane anisotropy). Figures 2(a) and 2(b) show 3D views of the magnetization vector trajectory for two special cases of pulse width, the first $[1.27 \mathrm{kA} / \mathrm{m}$ (16 Oe) and 400 ps FWHM] resulting in a half-precession quasi-ballistic switch, the second $[2.23 \mathrm{kA} / \mathrm{m}$ (28 Oe) and 730 ps FWHM] in a full-precession "nonswitch." Both trajectories begin with a small relaxive motion along the $y$ axis until the slew rate of the pulse rising edge is high enough to excite precessional motion. The torque induced by the cross product of $M$ and $H$ then tilts the vector up out of the $x-y$ plane. The resulting demagnetizing field (pointing straight down) then dominates the effective field contributions causing most of the precession to be around the $z$ axis. For the first case, the applied field decays after the magnetization crosses the $y-z$ plane resulting in a permanent switch. The field decay is timed to complete just as the vector reaches the $x-y$ plane $\left(m_{z}\right.$ becomes zero ${ }^{10}$ leading to this quasiballistic switching in which there are no residual precessional oscillations and completion is within 320 ps. With the pulse set at about $90 \%$ of the measured in-plane anisotropy field, these data are reminiscent of the macrospin ballistic switching calculated in Ref. 10 and correspond to a below-quasistaticthreshold demonstration of ballistic precessional switching. For the second case, the longer pulse causes one full oscillation around the $y$ axis, $m_{x}$ changing sign twice, and magnetization is left in the unswitched state at termination.

Further pulse width dependence is explored in Fig. 2(c) where an oscillation in the final state with increasing pulse width is seen. Each row is a vector plot versus time for the measured magnetization. The grayscale background is the magnetization angle computed from a macrospin simulation using the actual field pulse shapes. ${ }^{19}$ Complementary to Ref. 15 , the actual direction of the magnetization vector can be tracked and a direct comparison to simulation is possible.

The termination of the pulse determines whether the macrospin finishes in a switched state or not (that is, whether the field is removed after an odd or even number of half oscillations). For the first two rows, the vector does not cross the $y-z$ plane and no switching takes place. For the next three, the magnetization switches, indicated by both the white band and final direction of the arrows. Note the residual "ringing" in the third and fifth rows where termination is too early and too late, respectively. By the sixth row, the longer pulse has allowed a second crossing of the $y-z$ plane resulting in full precession and the no-switch final state. For the eighth-tenth rows, there are three crossings of the hard plane and 3/2oscillation switching. Simulation and experiment compare well with only row 8 differing in final state.

Interesting to note is that the final state (of the measured central spot location) in all the switched cases is metastably canted towards the $y$ axis and not aligned along the easy $x$ direction. This behavior has not been revealed in the previous works. In Ref. 14, vector resolved data were not presented for the switching case and the existence of the longitudinal biasing field would have complicated the interpretation anyway. In Ref. 15, the different method (high speed magnetoresistive measurement) lacks vector resolution and local probing capabilities; thus it is relatively insensitive 


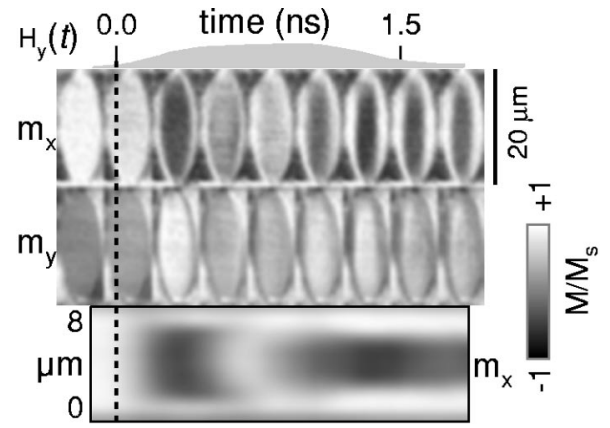

FIG. 3. Montage of spatial images (250 ps spacing) of the instantaneous magnetization during a precession that crosses the hard plane three times [also in EPAPS in movie form (Ref. 20)]. Frames 6-8 of $m_{x}$ show clear nonuniformity corresponding with decay of the field pulse (shown just above). Below is a horizontal linecut of $m_{x}$ displayed in grayscale as a function of time. A curvature (at 0.7 ns) indicates phase lag of the precession at the sample edges.

to the one-minus-cosine dependence for deviation from the easy axis and averages out any spatially dependent response. In both cases, macrospinlike response for precessional switching was heavily emphasized. However, the canting here suggests that even our sample is not a real macrospin but must have nonuniform spatial patterns in order to stabilize a local energy minimum. Further, the simulated data in Ref. 10, in going beyond the macrospin regime to the micromagnetic regime, show that structures of these sizes exhibit quite nonuniform and complex precessional switching processes. Both to explain this metastable canting and to truly explore precessional magnetization reversal, spatially resolved vector imaging becomes imperative.

Figure 3 shows a montage of grayscale images (250 ps intervals) of the instantaneous magnetization for a transverse pulse case of $3 / 2$ oscillations of the center of the structure. ${ }^{16}$ A thin band, about the thickness of the scalebar, around the perimeter of the ellipse cannot be accurately measured due to loss of signal as the laser spot encounters the sample edge. The magnetization seems uniform until frame 6 , where the pulse begins to turn off. With the Zeeman term removed, the $y$ component relaxes to zero and the vector turns to whichever easy axis is closer; the left and right edges align with the positive $x$ axis (white in $m_{x}$ ) while the center tilts toward the negative $x$ axis (black in $m_{x}$ ). The whole process is more easily seen in a vector movie. ${ }^{20}$ Thus, even though the precession is mostly coherent, because of the timing of the Zeeman energy removal, the magnetization has evolved to end in a substantially nonuniform state and the sample can clearly no longer be considered a macrospin.

In order to cause this large spatial inhomogeneity, the nonuniformity must have been developing before the release of the pulse field. Since the sample starts completely homogeneously, this implies a slight dephasing of the precessional oscillations across the sample. This dephasing further implies a spatial variation in internal field values between the edges and the center. The external magnetic field is essentially uniform throughout the volume of the sample (the small asymmetric $z$ gradient along the $y$ axis cannot account for the symmetric dephasing) finally implicating the demagnetizing

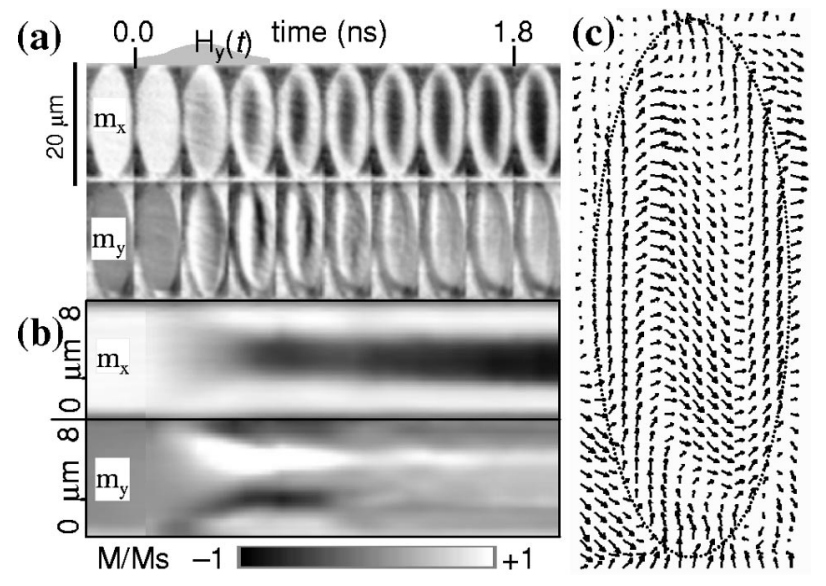

FIG. 4. (a) Montage of spatial images (200 ps spacing) of the magnetization response to coincident orthogonal magnetic field pulses. (b) Horizontal linecut vs time; $m_{y}$ exhibits complex variation. (c) Metastable state before slow field pulse shut-off. Magnetization eventually returns to positive saturation.

field as being inhomogeneous. Thus, despite the immersion magnetic layer and the elliptical shape, both of which should moderate (and homogenize) the demagnetizing field, magnetostatics still deteriorate this near-perfect macrospin.

These arguments can be understood more clearly viewing the grayscale linecut (horizontally across the sample) vs time shown below the montage. Starting from complete uniformity, a "C-shaped" curvature develops, becoming more pronounced over time. Response at the edges lags behind that at the center: a confirmation of the dephasing. Similar dephasing occurs for other pulse lengths with greater spatial variation tending for longer pulses (with more accompanying crossings of the hard plane). A brief examination of the lag indicates about a $15 \%$ difference in precessional frequencies corresponding to about a $30 \%$ difference in internal fields (ruling out a small variation in applied pulse field as the source). This difference should not lead to opposite polarity until after the third complete oscillation, however, here the phase lag is amplified by the termination of the pulse. In effect, these transverse, precession-inducing pulses can "transiently demagnetize" the sample.

This dephasing already occurs with only a single transverse field pulse. However, most bit selection schemes in MRAM require overlapping orthogonal pulses to address individual cells. Independently, each pulse is smaller than the switching threshold so that cells all along the word line and bit line are unaffected; combined, they switch the cell at the intersection of the two lines. To see if dephasing is intensified, the ellipse response to coincident crossing "half-select" pulses for this precessional mode switching is shown in Fig. 4. Several half-select combinations were tested ${ }^{21}$ and the amount of overlap of the two pulses was varied. One example is shown here. $H_{x}(t)$ is 15 ns long with a rise time of $2 \mathrm{~ns}$ and strength of $-500 \mathrm{~A} / \mathrm{m}(-6.3 \mathrm{Oe}) . H_{y}(t)$ is roughly Gaussian in shape, of peak amplitude $+716 \mathrm{~A} / \mathrm{m}$ ( $+9 \mathrm{Oe})$, with FWHM 360 ps, and is applied 4 ns after the onset of $H_{x}(t)$. Neither pulse causes a switch on its own; combined they should cause switching according to the StonerWohlfarth astroid. Ideally, the magnetization should coher- 
ently rotate to the hard plane and (i) back again, if no bias is present, or (ii) continue coherently to the new equilibrium if the longitudinal pulse is on.

Clearly, this is not the case here; the edges are rather fixed by the longitudinal field creating opposing polarity regions within half an oscillation period. More complicated yet, the $m_{y}$ response is split down the center of the element, the contrast implying clockwise rotation on the left half of the sample and counterclockwise rotation on the right; the magnetization begins precessing the wrong way in a sizable portion of the element. This perplexing response cannot be caused by the out-of-plane gradient of the pulse fields (related to the finite-size of the transmission line) since the symmetry breaking direction is opposite to what one would expect from this effect. It must be an energy-lowering demagnetizing maneuver by the element responding to some complex internal field (with differing areas even having opposite sign). The final, transiently demagnetized, metastable state is shown in Fig. 4(c). The center of the sample, having crossed the hard plane, is technically switched. However, it is only held metastably in place by the $-500 \mathrm{~A} / \mathrm{m}$ longitudinal field; some 5 or $10 \mathrm{~ns}$ after this field is removed, the center relaxes back to the initial unswitched direction. The large edge regions pull the center back and the permanent switch cannot be maintained. The transient demagnetization of the sample ultimately causes precessional mode coincident-pulse writing to fail in this case.
Though our sample is relatively large and well above the single domain limit, the failure here raises a warning flag for adopting precessional-style switching into MRAM and other devices. The fact that this failure appears in a device that closely emulates macrospinlike behavior ${ }^{14,15}$ compels reevaluation of recent optimistic perspectives. Clearly, the problem of dephasing and transient demagnetizing needs to become better understood, for both single and coincident pulse geometries of appropriate amplitudes and for samples of differing sizes, particularly down into the deep submicron regime. From a micromagnetic viewpoint, as long as a sample tolerates nonuniformities (that is, for all sizes above the single domain limit), one could expect dephasing.

In summary, spatially resolved precessional magnetic switching data have shown spatial incoherence during reversal. Dephasing of oscillations give evidence of nonuniform internal fields despite the homogenizing effects of both sample shape and embedding layer. The dephasing can lead to clear nonuniformity; with coincident magnetic field pulses, highly varying internal fields can cause complex magnetization response and metastable states. The consequence of such internal field deviation, transient demagnetization of the element, raises concern for the reliable use of precessional switching in sensor and MRAM applications.

The authors thank J. Das, R. Wirix-Speetjens, J. Bekaert, and P. Van Dorpe for technical assistance. W.K.H. acknowledges the financial support of NSERC (Canada). L.L. acknowledges the financial support of IWT (Belgium).
*Also at ESAT, K. U. Leuven, Leuven, Belgium.

${ }^{1}$ Magnetism III, edited by G. T. Rado and H. Suhl (Academic Press, New York, 1963), pp. 525-552.

${ }^{2}$ L. He et al., IEEE Trans. Magn. 30, 4086 (1994).

${ }^{3}$ W. K. Hiebert et al., Phys. Rev. Lett. 79, 1134 (1997).

${ }^{4}$ C. H. Back et al., Science 285, 864 (1999).

${ }^{5}$ M. Bauer et al., Phys. Rev. B 61, 3410 (2000).

${ }^{6}$ T. M. Crawford et al., Appl. Phys. Lett. 76, 2113 (2000).

${ }^{7}$ M. Bauer et al., Appl. Phys. Lett. 76, 2758 (2000).

${ }^{8}$ Y. Acremann et al., Science 290, 492 (2000).

${ }^{9}$ B. C. Choi et al., Phys. Rev. Lett. 86, 728 (2001).

${ }^{10} \mathrm{~J}$. Miltat et al., in Spin Dynamics in Confined Magnetic Structures $I$, edited by B. Hillebrands and K. Ounadjela (Springer, Berlin, 2002), Chap. 1.

${ }^{11}$ M. R. Freeman and W. K. Hiebert, Spin Dynamics in Confined Magnetic Structures I (Ref. 10), Ch. 4.

${ }^{12}$ W. K. Hiebert et al., Phys. Rev. B 65, 140404(R) (2002).

${ }^{13}$ S. Kaka and S. E. Russek, Appl. Phys. Lett. 80, 2958 (2002).

${ }^{14} \mathrm{Th}$. Gerrits et al., Nature (London) 418, 509 (2002).

${ }^{15}$ H. W. Schumacher et al., Phys. Rev. Lett. 90, 017204 (2003); 90, 017201 (2003).

${ }^{16}$ Attempts to measure from remanence, the dynamical response of the isolated Fig. 1(b) ellipse, resulted in highly complex and uninformative data.

${ }^{17}$ Figures $1(\mathrm{~b})$ and $1(\mathrm{c})$ are imaged via the time-resolved magnetooptic Kerr system in static mode with current lines as field sources. With the same, spot hysteresis loops are measured in situ on the embedded ellipse giving $H_{c}=1.07 \mathrm{kA} / \mathrm{m}$ and $H_{k}$ $\sim 1.43 \mathrm{kA} / \mathrm{m}$. The bulk unpatterned films were measured in an AGFM to give $H_{c} \sim 80 \mathrm{~A} / \mathrm{m}$ and $H_{k} \sim 400 \mathrm{~A} / \mathrm{m}$.
${ }^{18}$ Data were taken of the magnetization in the background material but the full implications of its behavior are not yet clear. More thorough work is needed to understand its effectiveness for different sized and shaped elements. However, starting from a nearperfect initial state represents the best situation as the motions are cleaner than they would be without the layer. We also assert that, due to its decreased thickness relative to the ellipse and because of the large angle motions induced on the ellipse by the field pulses, the effect of the layer on the ellipse once the dynamics start is probably a second order one.

${ }^{19}$ The parameters for the macrospin simulation are as follows: a saturation magnetization of $1.08 \mathrm{~T}$ is assumed, a damping parameter of 0.012 is derived from time-domain magneto-optic FMR tests on similar samples, an intrinsic uniaxial anisotropy of $H_{k}$ of $400 \mathrm{~A} / \mathrm{m}$ is assigned from bulk film measurements, and ellipsoidal demagnetizing factors of $0.0005,0.0014$, and 0.9981 corresponding to a sample size of $20 \mu \mathrm{m} \times 8 \mu \mathrm{m} \times 16 \mathrm{~nm}$, respectively, and shape anisotropy values of $400 \mathrm{~A} / \mathrm{m}, 1.12 \mathrm{kA} / \mathrm{m}$, and $859 \mathrm{kA} / \mathrm{m}$, respectively, are used.

${ }^{20}$ See EPAPS Document No. E-PRBMDO-68-R02326 for a vector movie of the data. A direct link to this document may be found in the online article's HTML reference section. The document may also be reached via the EPAPS homepage (http:// www.aip.org/pubservs/epaps.html) or from ftp.aip.org in the directory /epaps/. See the EPAPS homepage for more information.

${ }^{21}$ While some combinations caused temporary switching of the sample center (and some did not) none of the combinations led to permanent switching. In general, trapping of the long edges by the longitudinal field was exhibited. 